\title{
Scheduling in Mobile Ad Hoc Networks with Topology and Channel-State Uncertainty
}

\author{
Lei Ying \\ ECE Department \\ Iowa State University \\ Email: leiying@iastate.edu
}

\author{
Sanjay Shakkottai \\ ECE Department \\ The University of Texas at Austin \\ Email: shakkott@ece.utexas.edu
}

\begin{abstract}
We study throughput-optimal scheduling/routing over mobile ad-hoc networks with time-varying (fading) channels. Traditional back-pressure algorithms (based on the work by Tassiulas and Ephremides) require instantaneous network state (topology, queues-lengths, and fading channel-state) in order to make scheduling/routing decisions. However, such instantaneous network-wide (global) information is hard to come by in practice, especially when mobility induces a time-varying topology.

With information delays and a lack of global network state, different mobile nodes have differing "views" of the network, thus inducing uncertainty and inconsistency across mobile nodes in their topology knowledge and network state information. In such a setting, we first characterize the through-optimal rate region and develop a back-pressure-like scheduling algorithm, which we show is throughput-optimal. Then, by partitioning the geographic region spatially into disjoint tiles, and sharing delayed topology and network state information only among mobile nodes currently within each tile, we develop a localized low-complexity scheduling algorithm. The algorithm uses instantaneous local information (the queue length, channel state and current position at a mobile node) along with delayed network state information from nodes that were within its tile (i.e., from nodes that were within a nearby geographic region as opposed to network-wide information). The proposed algorithm is shown to be nearoptimal, where the geographic distance over which delayed network-state information is shared determines the provable lower bound on the achievable throughput.
\end{abstract}

\section{INTRODUCTION}

Mobile ad hoc networking is one of the most innovative emerging networking technologies and has broad applications in various domains (e.g., battlefield communications, search and rescue operations, range extension for rural networks). Mobile nodes communicate with each other using wireless communication, where simultaneous nearby transmissions can cause significant interference. To develop a high-performance mobile ad hoc network, a key step is to design scheduling algorithms that selectively activate a subset of links according to the known network state information in order to avoid excessive interference as well as maximize network throughput. In this paper, we study scheduling algorithms for mobile ad-hoc networks with time-varying (fading) channels. Most studies in literature that build on the on the work of Tassiulas and Ephremides [1], [2]) (in the context of time-varying channels and/or topology) require all nodes in the network to have (globally shared) instantaneous network state (e.g., topology knowledge, queues-lengths, and fading channel-state) in order to make scheduling/routing decisions. However, in a mobile network with a time-varying topology, it does not seem reasonable to expect all nodes to have such instantaneous network-wide (global) information. Furthermore, in general, there is no central controller in mobile ad hoc networks, so each mobile has to make transmission decisions based on the information it collects. Thus, a challenging problem is to develop distributed scheduling algorithms with channel and topology uncertainty.

We consider a network with $N$ sender-receiver (S-R) pairs, where the S-R pairs move according to Markovian processes. We assume that each mobile knows its own current position and instantaneous channel state, but it only has other mobiles' information with delay $\tau$. This information delay along with the lack of global network state induces uncertainty and inconsistency in the topology knowledge and network state information (due to the fact that different mobile nodes have different "views" of the network). Our focus of this paper is to first understand the fundamental network throughput region under the information inconsistency and topology uncertainty, and then develop online scheduling algorithms that are optimal or near optimal.

\section{A. Main Contributions}

The main contributions of this paper include:

(1) We first characterize the network throughput region under the information structure that each pair has own instantaneous channel and geographic information, but only other pairs' information with a delay of $\tau$ time slots.

(2) We then propose a back-pressure-like scheduling algorithm where each mobile first computes a locationbased threshold function with the global delayed information; and then makes transmission decisions based on its current position, instantaneous channel state and the threshold value. We show that the algorithm is throughput-optimal, i.e., it can stabilize the network as long as the traffic is within the network throughput region. Each mobile, however, needs to compute the threshold function, and the computation complexity is proportional to the network size.

(3) Finally, we propose a localized scheduling algorithm, where we partition the geographic region spatially into 
disjoint tiles and delayed topology and network state information are shared only among mobile nodes currently within each tile. We develop a near-optimal algorithm for distributed scheduling. The algorithm uses instantaneous local information along with delayed network state information from nodes that were within its tile (i.e., from nodes that were within a nearby geographic region as opposed to network-wide information). We show that this algorithm is near-optimal; more formally, we show that traffic $\boldsymbol{\lambda}[t]$ is supportable if $(1+\epsilon)(\boldsymbol{\lambda}[t]+\boldsymbol{\delta})$ is supportable under some throughput-optimal algorithm, where $\delta$ is the parameter depending on the spatial scale of the tiles. The computation complexity of the algorithm is only determined by the size of the tiles and the corresponding mobile nodes within a tile (as opposed to all mobile nodes in the network).

\section{B. Related Work}

Throughput-optimal routing/scheduling algorithm was first proposed in [1], [2]. Assuming that all mobile or static nodes have perfect global knowledge of the queue, channel and topology state, throughput-optimal routing/scheduling algorithms have been developed for different networks [3], [4], [5], [6], [7], [8], [9], [10], [11]. There has also been much work in developing distributed and low-complexity implementations [12], [13], [14], [15], [16], [17]. Please see [18], [19] for a survey.

There have been some studies in the context of incomplete network state information (missing/delayed channel, queue or topology state). To the best of our knowledge, the earliest work to consider delayed queue-length information and its impact on stability of back-pressure algorithms is [20]. In a down-link/up-link wireless scenario that explore the tradeoff between channel measurements and opportunistic gain, studies include [21], [22], [23], [24], [25], [26], [27], [28], [29], [30]. With i.i.d. channels and a static network, [31] has developed routing/scheduling algorithms with noisy channel estimates. In [32], the authors have studied throughput-optimal scheduling/routing in static ad hoc networks with delayed network state information (queue length and channel state). However, to the best of our knowledge, we are not aware of (near) throughput-optimal routing/scheduling results with limited and delayed channel/topology knowledge in a mobile context.

\section{Model AND Notations}

We consider a wireless network with $N$ sender-receiver (SR) pairs. We use $\mathcal{L}$ to denote the set of the S-R pairs. Without loss of generality, we assume the $N$ S-R pairs are deployed in a square area with side-length $Y$ as in Figure 1.

Traffic Model: We assume single-hop traffic in this paper, i.e., there is a traffic flow from sender $l$ to receiver $l$ for each $l \in \mathcal{L}$. We denote by $\lambda_{l}[t]$ the number of packets arriving at sender $l$ at time $t$. We assume that $\left\{\lambda_{l}[t]\right\}_{l \in \mathcal{L}}$ are stationary random variables, independent across different sender-receiver pairs, and bounded.

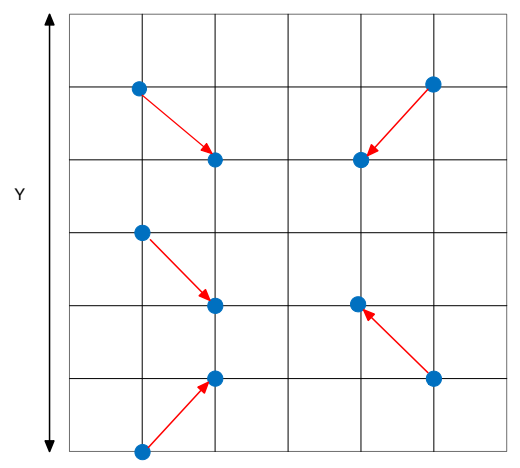

Fig. 1. $\quad$ S S-R pairs deployed in a square area with side-length $Y$

Mobility Model: We consider a discrete-time system. We assume that the pairs move at the beginning of each time slot, and stay still within a time slot. The mobility of each pair is Markovian and over a discrete square-lattice over the square region, i.e., the next location of a mobile is determined by its current location, and does not depend on the other history information, and the next location is on a (possibly fine resolution) grid. We assume that the distance a mobile can move at the beginning of a time slot is no more than $v_{\max }$. Furthermore, we assume that the mobility processes are stationary and ergodic, and the stationary distribution is uniform over the square area (we abuse notation in that the square area actually means the discrete-lattice over the square area). For simplicity, we also assume that the distance between a sender and its receiver is $D$, which is fixed for all $t$. Our results can be easily extended to the case where the distance between a sender and its receiver is time-varying, but upper and lower bounded. We denote by $S_{l}[t]$ the location of the sender $l$ at time slot $t$, and $R_{l}[t]$ the location of receiver $l$ at time slot $t$.

Channel Model: We assume that time-varying wireless channel between each S-R, and each channel can be characterized with a finite-state Markov chain. We denote by $C_{l}[t]$ the channel capacity of pair $l$ at time $t$, and assume $C_{l}[t] \leq C_{\max }$ for all $l \in \mathcal{L}$ and all $t$. Further, the channels are independent across sender-receiver pairs.

Queue Management: We assume that each sender maintains a queue, and the length of the queue at sender $l$ at time $t$ is denoted by $Q_{l}[t]$.

Information Set for Sender $l$ : We assume that at time $t$, sender $l$ has $\left\{C_{l}[s],\left(S_{l}[s], R_{l}[s]\right), Q_{l}[s]\right\}$ for $s \leq t$, and $\left\{C_{j}[s],\left(S_{j}[s], R_{j}[s]\right), Q_{j}[s]\right\}$ for all other $j \in \mathcal{L}$ and all $s \leq$ $t-\tau$, where $\tau$ is the delay.

Scheduling-Decision Vector: We define a vector $\mathbf{A}[t]$ to be the scheduling-decision vector at time $t$ such that $A_{l}[t]=1$ if the sender $l$ transmits at time $t$; and $A_{l}[t]=0$ otherwise. Note that $A_{l}[t]$ is a function of the information available to sender $l$.

Location-Based Threshold Scheduling: We study a class of scheduling policies which we denote as locationbased threshold scheduling policies. A location-based thresh- 
old scheduling policy is defined by a real-valued function $\Theta_{l}\left(s_{l}, r_{l}\right)$, which depends on the mobile's current location for given delayed channel and queue length information available at the sender (please see Section III-C for a more precise description). If sender $l$ is at location $\left(s_{l}, r_{l}\right)$ and the channel state $c_{l} \geq \Theta_{l}\left(\left(s_{l}, r_{l}\right)\right)$, then sender $l$ will transmit. We further define $\boldsymbol{\Theta}(\mathbf{s}, \mathbf{r})=\left\{\Theta_{l}\left(s_{l}, r_{l}\right)\right\}_{l}$.

Interference Model: We assume a geographic-based collision model in this paper. If two links interfere with each other, simultaneous transmissions on the two links will lead to a collision and no information (packet) can get through. Consider pair $l$, the transmission of pair $j$ will interfere with the transmission of pair $l$ if $\left|S_{j}[t]-R_{l}[t]\right| \leq(1+\Delta) D$, where $\left|S_{j}[t]-R_{l}[t]\right|$ is the Euclidean distance, and $\Delta$ is the a protocol specified guard-zone to prevent interference.

Note that $\mathbf{C}[t]=\left\{C_{l}[t]\right\}_{l \in \mathcal{L}}, \mathbf{S}[t]=\left\{S_{l}[t]\right\}_{l \in \mathcal{L}}$ and $\mathbf{R}[t]=$ $\left\{R_{l}[t]\right\}_{l \in \mathcal{L}}$ are random variables, and we assume that they are independent.

\section{Throughput-Optimal Scheduling Algorithm WITH TOPOLOGY UNCERTAINTY}

In this section, we first characterize the network throughput region under channel and topology uncertainty.

\section{A. Optimal Throughput Region}

It is easy to see that the transmission rates of the pairs at time $t$ are determined by the following three parameters: (i) channel condition $\mathbf{C}[t]$, (ii) network topology, which is defined by the mobiles' positions $(\mathbf{S}[t], \mathbf{R}[t])$, and (iii) the scheduling decision $\mathbf{A}[t]$.

Now assume that $\mathbf{C}[t]=\mathbf{c},(\mathbf{S}[t], \mathbf{R}[t])=(\mathbf{s}, \mathbf{r})$, and $\mathbf{A}[t]=\mathbf{a}$ (we use lower-cases to denote the realizations of random variables). Under the collision-model defined in Section II, a maximum link rate $c_{l}$ can be achieved over link $l$ if there is no other active pairs interfering pair $l$; otherwise, the link rate is zero. Mathematically, we can define a link-rate vector $L_{\{\mathbf{c},(\mathbf{s}, \mathbf{r}), \mathbf{a}\}}$ such that

$$
L_{\{\mathbf{c},(\mathbf{s}, \mathbf{r}), \mathbf{a}\}, l}=c_{l}
$$

if $a_{h}=0$ for any pair $h$ such that $\left|s_{h}-r_{l}\right| \leq(1+\Delta)\left|s_{l}-r_{l}\right|$; and

$$
L_{\{\mathbf{c},(\mathbf{s}, \mathbf{d}), \mathbf{a}\}, l}=0
$$

otherwise.

Given the delayed information

$$
\{\mathbf{C}[t-\tau],(\mathbf{S}[t-\tau], \mathbf{R}[t-\tau])\}=\{\mathbf{c},(\mathbf{s}, \mathbf{r})\},
$$

we define $\Lambda_{\{\mathbf{c},(\mathbf{s}, \mathbf{r})\}}$ such that

$$
\begin{aligned}
& \Lambda_{\{\mathbf{c},(\mathbf{s}, \mathbf{r})\}} \\
= & \mathcal{C} \mathcal{H}_{\boldsymbol{\Theta}}\left(\mathbf { E } \left[\mathbf{L}_{\left\{\mathbf{C}[t],(\mathbf{S}[t], \mathbf{R}[t]), \mathbf{1}_{\mathbf{C}[t] \geq \Theta(\mathbf{S}[t], \mathbf{R}[t])} \mid\right.} \mid\right.\right. \\
& \mathbf{C}[t-\tau],(\mathbf{S}[t-\tau], \mathbf{R}[t-\tau])=\{\mathbf{c},(\mathbf{s}, \mathbf{r})\}]),
\end{aligned}
$$

where

$$
\mathbf{1}_{\mathbf{C}[t] \geq \Theta(\mathbf{S}[t], \mathbf{R}[t])}=\left\{1_{C_{l}[t]>\Theta_{l}\left(S_{l}[t], R_{l}[t]\right)}\right\}_{l} .
$$

Note that $\Lambda_{\{\mathbf{c},(\mathbf{s}, \mathbf{r})\}}$ is independent of $t$ because both $\mathbf{C}[t]$ and $(\mathbf{S}[t], \mathbf{R}[t])$ are assumed to be stationary random variables. Finally, we define

$$
\begin{array}{r}
\Lambda_{\tau}=\left\{\boldsymbol{\lambda}: \boldsymbol{\lambda}=\sum_{\{\mathbf{c},(\mathbf{s}, \mathbf{r})\}} \pi_{\{\mathbf{c},(\mathbf{s}, \mathbf{r})\}} \eta_{\{\mathbf{c},(\mathbf{s}, \mathbf{r})\}},\right. \\
\left.\eta_{\{\mathbf{c},(\mathbf{s}, \mathbf{r})\}} \in \Lambda_{\{\mathbf{c},(\mathbf{s}, \mathbf{r})\}}\right\},
\end{array}
$$

where $\pi_{\{\mathbf{c},(\mathbf{s}, \mathbf{r})\}}$ is the stationary distribution of $\{\mathbf{C}[t],(\mathbf{S}[t], \mathbf{R}[t])\}=\{\mathbf{c},(\mathbf{s}, \mathbf{r})\}$

Given traffic $\boldsymbol{\lambda}[t]$, we say the traffic is within the network throughput region (or the traffic is supportable) if there exists a scheduling under which $\mathbf{E}\left[Q_{l}[t]\right] \leq Q_{\max }$ for all $l$ and all $t$, where $Q_{\max }$ is some positive number.

In the following theorem, we prove that $\Lambda_{\tau}$ is the network throughput region.

Theorem 1: Traffic $\boldsymbol{\lambda}[t]$ is supportable if and only if $\mathbf{E}[\boldsymbol{\lambda}[t]] \in \Lambda_{\tau}$.

Remark: The key idea to prove the theorem above is to show that if there exists a scheduling policy which can support the traffic, then we can construct a corresponding locationbased threshold policy under which the mean queue lengths are also bounded. The theorem then follows since the servicerate vector under any location-based threshold policy is in the set $\Lambda_{\tau}$. The proof is similar to the analysis in [32], and is omitted due to page limitation.

Next we use a simple example to illustrate $\mathbf{L}_{\{\mathbf{c},(\mathbf{s}, \mathbf{d}), \mathbf{a}\}}$ and throughput-region $\Lambda_{\tau}$.

\section{B. An Illustrative Example}

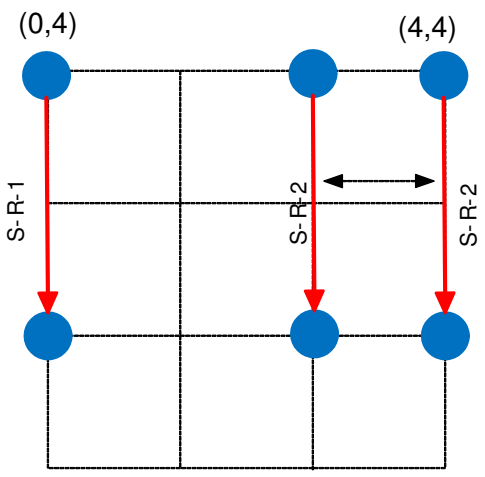

$(0,0)$

$(4,0)$

Fig. 2. Mobile ad hoc network example

Consider a simple example with two S-R pairs (S-R-1 and S-R-2) as in Figure 2, where S-R-1 is not mobile and located at $((0,4),(0,1))$, and S-R-2 moves between locations $((3,4),(3,1))$ and $((4,4),(4,1))$ respectively. Assume that link capacities are unity for both of the links, i.e., $C_{1}[t]=$ $C_{2}[t]=1$ (in other words, there is no channel fading). However, the two pairs interfere with each other when S-R-2 is at location $((3,4),(3,1))$. The mobility of S-R-2 follows a Markov process as shown in Figure 3. 


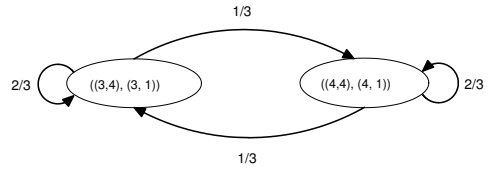

Fig. 3. Markovian mobility

Note that when S-R-2 at position $((3,4),(3,1))$, the two pairs cannot be active (i.e., successfully transmit packets) simultaneously. Thus, we have

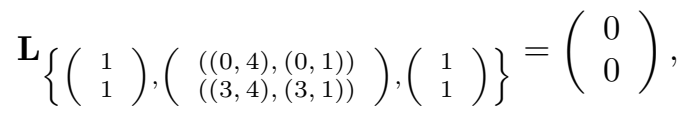

and

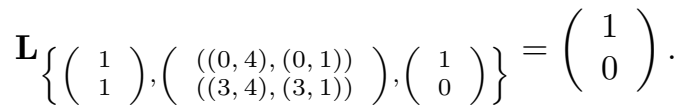

Next we assume that the information delay $\tau=1$. Since both channels are time-invariant and the position of S-R-1 is fixed, the information S-R-1 has is completely determined by the delayed information from S-R2 . It is easy to verify that given $\left(S_{2}[t-1], R_{2}[t-1]\right)=$ $((3,4),(3,1)), \Lambda_{\left\{\left(\begin{array}{l}1 \\ 1\end{array}\right),\left(\begin{array}{c}((0,4),(0,1)) \\ ((3,4),(3,1))\end{array}\right)\right\}}$ is as shown in Figure 4(a); and given $\left(S_{2}[t-1], R_{2}[t-1]\right)=((4,4),(4,1))$, $\Lambda_{\left\{\left(\begin{array}{l}1 \\ 1\end{array}\right),\left(\begin{array}{l}((0,4),(0,1)) \\ ((4,4),(4,1))\end{array}\right)\right\}}$ is as in shown Figure 4(b).

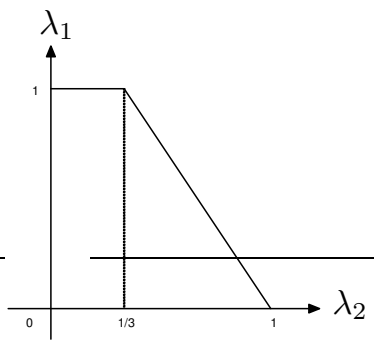

(a) Case 1

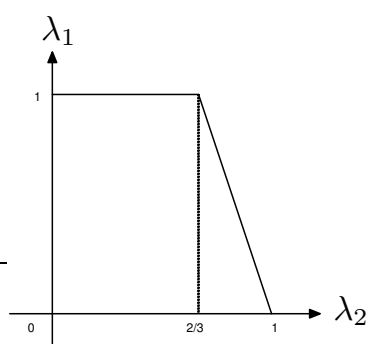

(b) Case 2
Fig. 4. Throughput regions under different delayed information

Note that

$$
\begin{aligned}
& \operatorname{Pr}\left(\left(S_{2}[t], R_{2}[t]\right)=((3,4),(3,1))\right) \\
= & \operatorname{Pr}\left(\left(S_{2}[t], R_{2}[t]\right)=((4,4),(4,1))\right) \\
= & \frac{1}{2}
\end{aligned}
$$

so the throughput region $\Lambda_{\tau=1}$ is as shown in Figure 5. The throughput region without any information delay is also shown in Figure 5 using the dotted line as a comparison. We can see that the throughput region shrinks when we have delays in topology/channel knowledge.

\section{Throughput-Optimal Scheduling Algorithm}

In this section, we propose a throughput-optimal scheduling algorithm which stabilizes the network for $\boldsymbol{\lambda}[t]$ within the network throughput region.

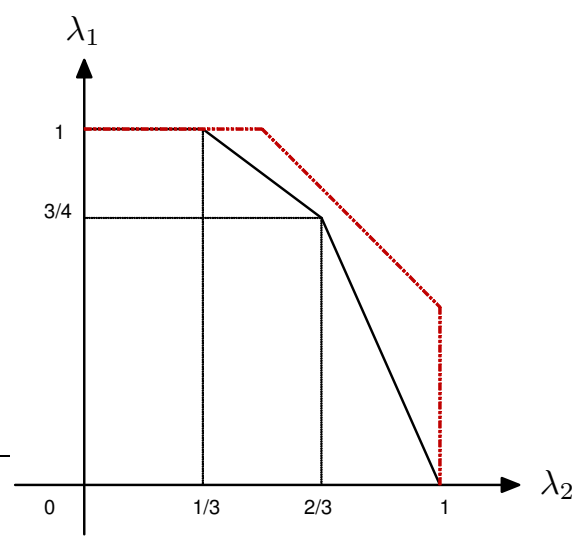

Fig. 5. Throughput region $\Lambda_{\tau=1}$

Threshold-Based Scheduling: Given the delayed information $\mathbf{Q}[t-\tau]$ and

$$
\mathbf{J}[t-\tau]:=\{\mathbf{C}[t-\tau],(\mathbf{S}[t-\tau], \mathbf{R}[t-\tau])\},
$$

which is available at all senders, the senders make transmission decisions according to the following two steps:

Step 1: Each sender computes a set of threshold function $\Theta_{l}^{*}\left(S_{l}[t], R_{l}[t]\right)$ (for all $l \in \mathcal{L}$ ) that solves the following optimization problem.

$$
\begin{gathered}
\max \sum_{l \in \mathcal{L}} Q_{l}[t-\tau] \mathbf{E}\left[C_{l}[t] 1_{C_{l}[t] \geq \Theta_{l}\left(S_{l}[t], R_{l}[t]\right)} \times\right. \\
\prod_{j \neq l}\left(\begin{array}{cc}
1-1 & \left.\left.\begin{array}{c}
S_{j}[t]-R_{l}[t] \mid \leq(1+\Delta) D, \\
C_{j}[t] \geq \Theta_{j}\left(S_{j}[t], R_{j}[t]\right)
\end{array}\right) \mid \mathbf{J}[t-\tau]\right] .
\end{array}\right.
\end{gathered}
$$

Note that $(\mathbf{S}[t], \mathbf{R}[t])$ is random variable in this calculation, i.e., the instantaneous geographic information is not used in computing the threshold function.

Step 2: Sender $l$ transmits with rate $C_{l}[t]$ if its current location is $\left(S_{l}[t], R_{l}[t]\right)$ and

$$
C_{l}[t] \geq \Theta_{l}^{*}\left(S_{l}[t], R_{l}[t]\right) .
$$

The following theorem shows that the algorithm proposed above is throughput optimal.

Theorem 2: Given a traffic $\boldsymbol{\lambda}[t]$ such that $(1+\epsilon) \mathbf{E}[\boldsymbol{\lambda}[t]] \in$ $\Lambda_{\tau}$, the network is stochastically stable under the thresholdscheduling algorithm.

Proof: Define a Lyapunov function

$$
V[t]=\sum_{l \in \mathcal{L}} Q_{l}^{2}[t]
$$

The key idea is to prove that

$\mathbf{E}[V[t+1]-V[t] \mid \mathbf{Q}[t-\tau]] \leq-2 \epsilon \sum_{l \in \mathcal{L}} \mathbf{E}\left[\boldsymbol{\lambda}_{l}[t]\right] Q_{l}[t-\tau]+B$,

for some positive $B$. The detail proof is provided in Appendix A.

Remark: Since the delayed information $\mathbf{Q}[t-\tau]$ and $\mathbf{J}[t-\tau]$, and channel state distributions are available at all mobiles, each 
mobile can solve the optimization problem (2), and makes their transmission decisions based on its current location, instantaneous channel state, and the threshold value. Thus, the algorithm is a distributed algorithm. However, the optimization problem (2) involves network-wide delay information, so the complexity is at the scale as the network size, which could be very high when the network size is large. In the next section, we will develop a low-complexity (where the decision depends only on "local" delayed information) and near optimal implementation.

\section{LOW-COMPLEXITY AND NEAR-OPTIMAL IMPLEMENTATION}

In this section, we propose a scheduling algorithm whose information and computation complexity is independent of the network size. The idea is to partition the geographic region spatially into disjoint tiles and only share delayed topology and network state information among mobile nodes within each tile. Then the computation complexity is determined by the size of the tiles.

We first assume that each mobile is equipped with a GPS or appropriate technology (e.g., cell tower based triangulation), so the mobiles have knowledge of their geographic locations. We partition the square area into tiles with side-length $2 W+X$ as shown in Figure 6, where $W$ and $X$ are positive numbers and

$$
W \geq \max \left\{\tau v_{\max },(1+\Delta) D\right\} .
$$

Each tile is further separated into two areas as shown in Figure 6: (i) active-area: a square with side-length $X$ centered at the corresponding square-cell; (ii) inactive-area: the area outside the active-area.

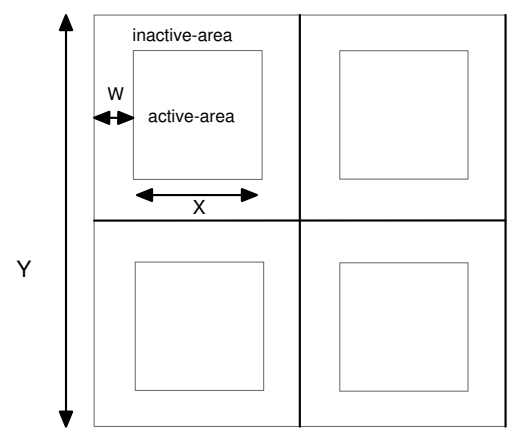

Fig. 6. Low complexity implementation

The tile-partition information is assumed to be known by mobiles so that the mobiles can determine their current tile and type (active or inactive). We consider scheduling schemes such that senders (transmitters) within an inactive-area keep silent (i.e., do not transmit), which guarantees that transmissions within different active-areas will not interfere with each other (this follows because the inactive area is "wider" than the radio range).

Also, we observe that the mobiles in an active-area at time $t$ must be in the tile containing the active-area at time $t-\tau$.
This follows directly from (3), and the fact that a mobile can move a distance no more than $v_{\max }$ per time slot.

We use $z$ to denote the index of a tile, $z^{a}$ to indicate the active-area in tile $z$, and $z^{i}$ to indicate the inactive-area in tile $z$. Furthermore, let $\mathcal{C}_{z}[t]$ denote the set of senders in the cell $z$. As explained, we have

$$
\mathcal{C}_{z^{a}}[t] \subseteq \mathcal{C}_{z}[t-\tau] .
$$

Thus the senders who are in the same active-area at time $t$ were in the same tile at time $t-\tau$. We also note that since there is no transmission in the inactive-area, the transmissions in different active-areas do not interfere. Thus, we can decompose the network-wide optimization problem into those corresponding to individual tiles, and the delayed state information only needs to be shared within tiles.

Recall that mobiles know which tile they are located in. The topology and channel information are exchanged among mobile nodes within each tile with delay $\tau$. The queue-length information is also assumed to be periodically exchanged with delay $\tau_{q}$, and we assume that $\tau_{q} \geq \tau$.

In this setting, we propose the following localized scheduling algorithm.

Localized Threshold-Based Scheduling: At tile $z$, the mobiles know $Q_{l}\left[t-\tau_{q}\right]$ and $\left\{C_{l}[t-\tau],\left(S_{l}[t-\tau], R_{l}[t-\tau]\right)\right\}$ for all $l$ such that $S_{l}[t-\tau] \in \mathcal{C}_{z}[t-\tau]$. The senders then make transmission decisions according to the following two steps:

Step 1: Sender $l$ computes a set of threshold function $\left.\hat{\Theta}_{l} \overline{\left(S_{l}[t], R_{l}\right.}[t]\right)$ that solves the following optimization problem.

$$
\begin{gathered}
\max \sum_{\mathcal{C}_{z}: S_{l}[t] \in C_{z}} Q_{j}[t-\tau] \mathbf{E}\left[C_{j}[t] 1_{C_{j}[t] \geq \Theta_{j}\left(S_{j}[t], R_{j}[t]\right)} \times\right. \\
\prod_{k \neq j}\left(\begin{array}{c}
\left.1-1 \quad \begin{array}{c}
\left|S_{k}[t]-R_{j}[t]\right| \leq(1+\Delta) D, \\
C_{k}[t] \geq \Theta_{k}\left(S_{k}[t], R_{k}[t]\right)
\end{array}\right) \mid \mathbf{J}_{z}[t-\tau] \\
\quad
\end{array}\right],
\end{gathered}
$$

where

$$
\mathbf{J}_{z}[t-\tau]=\left\{C_{j}[t-\tau],\left(S_{j}[t-\tau], R_{j}[t-\tau]\right)\right\}_{j \in \mathcal{C}_{z}},
$$

and the threshold function needs to satisfy that $\hat{\Theta}\left(S_{j}[t], R_{j}[t]\right)=\infty$ if $S_{j}[t] \notin \mathcal{C}_{z^{a}}$ (sender $j$ needs to keep silent if it is in an inactive-area).

Step 2: Sender $l$ transmits with rate $C_{l}[t]$ if its current location is $\left(S_{l}[t], R_{l}[t]\right)$ and

$$
C_{l}[t] \geq \hat{\Theta}_{l}\left(S_{l}[t], R_{l}[t]\right) .
$$

Recall that the location of a mobile is uniformly distributed over the square domain. Thus, the fraction of time a mobile is positioned in inactive-areas is by

$$
\frac{(2 W+X)^{2}-X^{2}}{(2 W+X)^{2}}=\quad 1-\left(\frac{X}{2 W+X}\right)^{2} .
$$

Further, since the link capacity is upper bounded by $C_{\max }$, the throughput-loss because of a mobile moving into inactiveareas is upper bounded by $C_{\max }\left(1-\left(\frac{1}{2 \frac{W}{X}+1}\right)^{2}\right)$, which goes to zero when $W / X \rightarrow 0$. 

that

In the next theorem, we prove that given a traffic $\boldsymbol{\lambda}[t]$ such

$$
(1+\epsilon)\left(\mathbf{E}[\boldsymbol{\lambda}[t]]+C_{\max }\left(1-\left(\frac{1}{2 \frac{W}{X}+1}\right)^{2} \mathbf{1}\right)\right) \in \Lambda_{\tau}
$$

where 1 is an $N$ identity vector, then the network is stochastically stable under the localized threshold-based scheduling.

Theorem 3: Given

$$
(1+\epsilon)\left(\mathbf{E}[\boldsymbol{\lambda}[t]]+C_{\max }\left(1-\left(\frac{1}{2 \frac{W}{X}+1}\right)^{2}\right) \mathbf{1}\right) \in \Lambda_{\tau},
$$

the network is stochastically stable under the localized threshold-based scheduling algorithm.

Proof: To prove the theorem, the idea is to consider a virtual network, where when sender $l$ is in an inactive-area, the arrivals is $\lambda_{l}[t]+C_{\max }$, and sender $l$ can transmit with rate $C_{\max }$ without causing any interference to other pairs.

Consider a modified localized threshold-based scheduling, which is the same as the original algorithm except that $\Theta_{l}\left(S_{l}[t], R_{l}[t]\right)=0$ (instead of $\Theta_{l}\left(S_{l}[t], R_{l}[t]\right)=\infty$ ). It is easy to see that the queue-evolution of the virtual network under the modified algorithm is the same as the original network. Thus, we analyze the virtual network with arrival $\lambda[t]$.

Given $\mathbf{Q}\left[t-\tau_{q}\right]$ and $\{\mathbf{C}[t-\tau],(\mathbf{S}[t-\tau], \mathbf{R}[t-\tau])\}$, let $\mathbf{\Theta}^{*}$ denote the optimal solution to (2), and $\tilde{\boldsymbol{\Theta}}$ denote the threshold function of the modified algorithm. First, for an active area $\mathcal{C}_{z^{a}}$, we have

$$
\begin{aligned}
& \sum_{l: \mathbf{S}_{l}[t] \in \mathcal{C}_{z^{a}}} Q_{l}\left[t-\tau_{q}\right] \mathbf{E}\left[C_{l}[t] 1_{C_{l}[t] \geq \tilde{\Theta}_{l}\left(S_{l}[t], R_{l}[t]\right)} \times\right. \\
& \left.\prod_{j \neq l}\left(\begin{array}{cc}
1-1 & \\
& \left.\mid S_{j}[t]-R_{l}[t]\right] \leq(1+\Delta) D, \\
C_{j}[t] \geq \Theta_{j}\left(S_{j}[t], R_{j}[t]\right)
\end{array}\right) \mid \mathbf{J}[t-\tau]\right] \\
& \geq \sum_{l: \mathbf{S}_{l}[t] \in \mathcal{C}_{z^{a}}} Q_{l}\left[t-\tau_{q}\right] \mathbf{E}\left[C_{l}[t] 1_{C_{l}[t] \geq \Theta_{l}^{*}\left(\mathbf{S}_{l}[t], \mathbf{R}_{l}[t]\right)} \times\right. \\
& \left.\prod_{j \neq l}\left(\begin{array}{cc}
1-1 & \\
& \left|S_{j}[t]-R_{l}[t]\right| \leq(1+\Delta) D, \\
C_{j}[t] \geq \Theta_{j}^{*}\left(S_{j}[t], R_{j}[t]\right)
\end{array}\right) \mid \mathbf{J}[t-\tau]\right]
\end{aligned}
$$

Next for an inactive area $\mathcal{C}_{z^{i}}$, we have

$$
\begin{aligned}
& \sum_{l: S_{l}[t] \in \mathcal{C}_{z^{i}}} Q_{l}\left[t-\tau_{q}\right] \mathbf{E}\left[C_{l}[t] 1_{C_{l}[t] \geq \tilde{\Theta}_{l}\left(S_{l}[t], R_{l}[t]\right)} \times\right. \\
= & \prod_{j \neq l}\left(\begin{array}{cc}
1-1 \\
\left.\begin{array}{c}
\left.\mid S_{j}[t]-R_{l}[t]\right] \leq(1+\Delta) D \\
C_{j}[t] \geq \tilde{\Theta}_{j}\left(S_{j}[t], R_{j}[t]\right)
\end{array}\right) \mid \mathbf{J}[t-\tau]
\end{array}\right] \\
\geq & \sum_{l: S_{l}[t] \in \mathcal{C}_{z^{a}}} Q_{l}\left[t-\tau_{q}\right] C_{\max } \\
& \prod_{l: S_{l}[t] \in \mathcal{C}_{z^{i}}} Q_{l}\left[t-\tau_{q}\right] \mathbf{E}\left[C_{l}[t] 1_{C_{l}[t] \geq \Theta_{l}^{*}\left(S_{l}[t], R_{l}[t]\right)} \times\right. \\
& \prod_{j \neq l}\left(\begin{array}{c}
1-1 \\
\left.\mid \begin{array}{c}
\left|S_{j}[t]-R_{l}[t]\right| \leq(1+\Delta) D, \\
C_{j}[t] \geq \Theta_{j}^{*}\left(S_{j}[t], R_{j}[t]\right)
\end{array}\right) \mid \mathbf{J}[t-\tau]
\end{array}\right]
\end{aligned}
$$

Inequalities (5) and (6) imply value of (2) under the virtual network is larger than the one under the original network. This is the key fact and can be used to prove theorem. The detailed proof is provided in Appendix B.

\section{Simulations}

In this section, we further study the performance of our algorithms using simulations. We consider a network as shown in Figure 7, in which there are four sender-receiver pairs. At beginning of each time slot, an S-R pair moves to its left or right with equal probability if the pair is not at the boundary of the network. Otherwise (at the network boundary), the S-R pair moves away from the boundary with probability $1 / 2$, or stays at the current position with probability $1 / 2$.

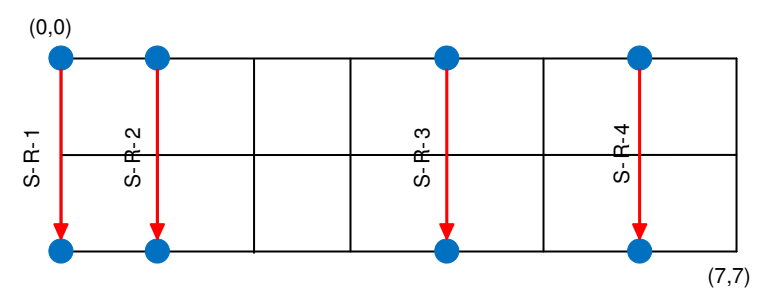

Fig. 7. Network used in simulations

We assume that two transmissions collide if the senders are at the same location or next to each other. We further assume the link rates are one packet per time slot for all links and all time, and the information delay $\tau=1$. The arrivals are assumed to be Bernoulli arrivals with rate $\lambda_{1}=\lambda_{2}=\lambda_{3}=$ $\lambda_{4}=\lambda$.

\section{A. Threshold-based scheduling algorithm versus the mismatch algorithm}

We first compare the performance of threshold-based scheduling algorithm with an algorithm where the senders treat the most recent information they have as instantaneous information (i.e., they ignore the fact that it is delayed) and make scheduling decisions based on the back-pressure algorithm. Note that the back-pressure algorithm is throughput optimal when global instantaneous information is used. The algorithm is named as the mismatch scheduling algorithm because the information the senders use is different from that for which the algorithm was designed for.

Mismatch Scheduling Algorithm: Given the delayed information $\mathbf{Q}[t-\tau]$ and

$$
\mathbf{J}[t-\tau]:=\{\mathbf{C}[t-\tau],(\mathbf{S}[t-\tau], \mathbf{R}[t-\tau])\},
$$

which is available at all senders, the senders make transmission decisions according to the following two steps:

Step 1: Sender $l$ computes decision vector $\tilde{\mathbf{A}}^{l, *}$ that solves the following optimization problem:

$$
\max _{\tilde{\mathbf{A}}^{l}} \sum_{j} \tilde{Q}_{j}^{l}[t] \tilde{C}_{j}^{l}[t] \tilde{A}_{j}^{l} \prod_{k \neq j}\left(1-\tilde{A}_{k}^{l} 1_{\left|\tilde{S}_{k}^{l}[t]-\tilde{R}_{j}^{l}[t]\right| \leq(1+\Delta) D}\right),
$$

where $(\cdot)_{j}^{l}[t]=(\cdot)_{j}[t]$ if $j=l$; otherwise $(\cdot)_{j}^{l}[t]=(\cdot)_{j}[t-\tau]$. Note that sender $l$ has its own instantaneous channel-state, 
location, and queue information, and delayed information of other pairs (which are all however treated as instantaneous information by sender $l$ in this algorithm).

Step 2: Sender $l$ transmits if $\tilde{A}_{l}^{l, *}=1$.

We choose $\lambda=0.20,0,21,0.22, \ldots, \lambda_{\max }$, where $\lambda_{\max }$ is the arrival rate under which the network is critically loaded (we say the network is critically loaded if the average queue lengths are larger than 100). We executed the simulation for $10^{5}$ iterations under both algorithms, and computed the average queue-lengths. The results are shown in Figure 8, which includes the average queue-lengths of S-R-1 for different values of $\lambda$ (similar results hold for other sender-receiver pairs).

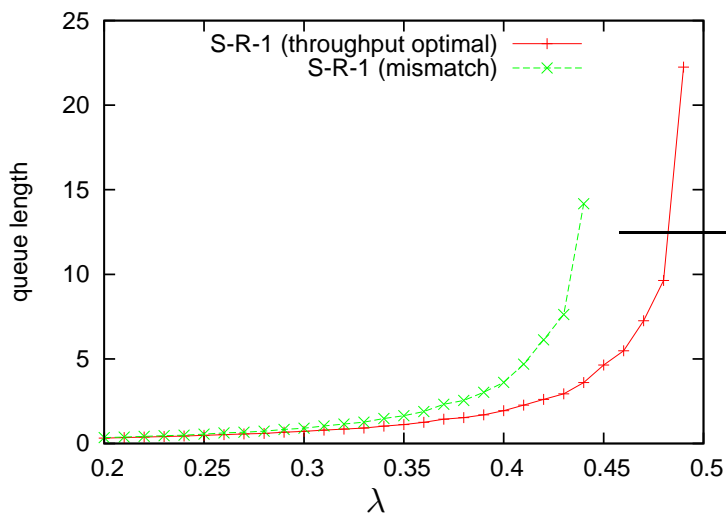

Fig. 8. Threshold-based algorithm $v s$ the mismatch algorithm

From Figure 8, we can see that the threshold-based scheduling algorithm (which is throughput-optimal) has smaller average queue-length. We also have that $\lambda_{\max }=0.49$ under the threshold-based algorithm, and $\lambda_{\max }=0.44$ under the mismatch algorithm, i.e., the threshold-based algorithm yields more than $10 \%$ throughput improvement.

\section{B. Localized threshold-based algorithm versus localized mis- match algorithm}

We divide the network into two subnetworks as shown in Figure 9, and compare the performance of the localized threshold-based scheduling algorithm with a localized mismatch algorithm under this tiling. The localized mismatch algorithm is an algorithm where sender $l$ only has delayed information of the pairs in the same subnetwork (tile). The scheduling decision is computed similar to the mismatch algorithm with the delayed information limited to the subnetwork (tile) the sender $l$ is in.

We choose $\lambda=0.20,0.21,0.22, \ldots, \lambda_{\max }$. For each $\lambda$, we executed the simulation for $10^{5}$ iterations under both algorithms, and computed the average queue-lengths. The results are shown in Figure 10. Again, we can see that the localized threshold-based scheduling algorithm yields a smaller average queue-length. In this simulation, $\lambda_{\max }=0.40$ under the localized threshold-based scheduling, and $\lambda_{\max }=0.36$ under the localized mismatch algorithm.

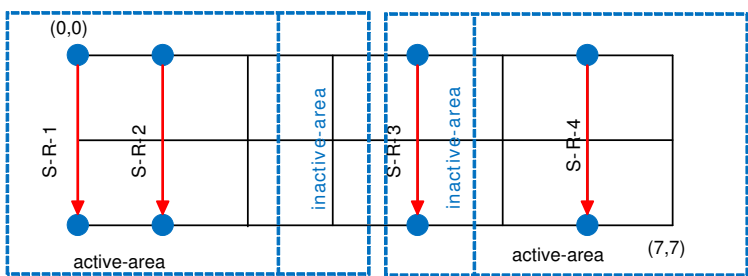

Fig. 9. Network partition

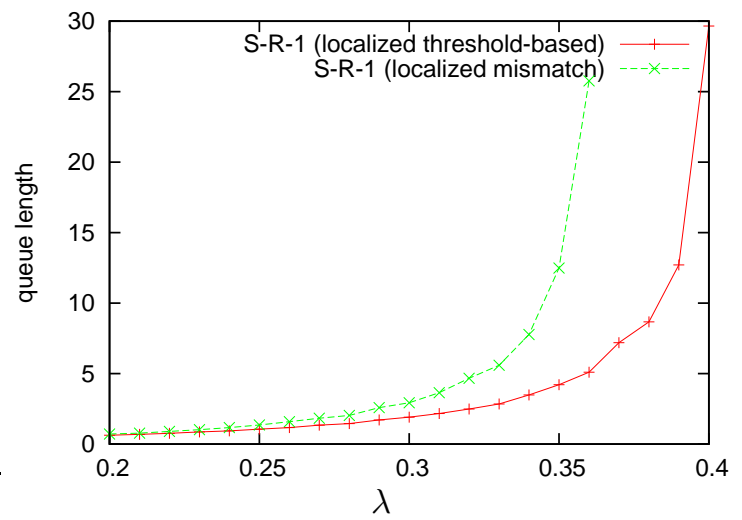

Fig. 10. Localized threshold-based algorithm vs localized mismatch algorithm

From the simulations above, we can see that properly exploiting delayed information can improve the network throughput and reduce the backlogs.

\section{CONCLUSION}

In this paper, we studied throughput-optimal routing/scheduling for mobile ad hoc networks with information delays. We characterized the network throughput region under channel and topology uncertainty. We also proposed a scheduling algorithm where the scheduling decisions are made based each mobile's instantaneous information and delayed information from local geographic regions. Future directions of this research include: (i) considering multi-hop traffic flows instead of only peer-to-peer communications; and (ii) considering physical interference model and design joint power control and scheduling algorithms.

Acknowledgment: This work was partially supported by NSF Grants CNS-0347400, CNS-0519535, CNS-0721380, and CNS-0831756, the Darpa ITMANET program and the DTRA grant HDTRA1-08-1-0016.

\section{REFERENCES}

[1] L. Tassiulas and A. Ephremides, "Stability properties of constrained queueing systems and scheduling policies for maximum throughput in multihop radio networks," IEEE Trans. Automat. Contr., vol. 4, pp. 1936-1948, December 1992

[2] - "Dynamic server allocation to parallel queues with randomly varying connectivity," IEEE Trans. Inform. Theory, vol. 39, pp. 466478, March 1993.

[3] M. Andrews, K. Kumaran, K. Ramanan, A. Stolyar, R. Vijayakumar, and P. Whiting, "CDMA data QoS scheduling on the forward link with variable channel conditions," Bell Labs Tech. Memo, April 2000. 
[4] A. Stolyar and K. Ramanan, "Largest weighted delay first scheduling: Large deviations and optimality," Adv. Appl. Prob., vol. 11, pp. 1-48, 2001.

[5] S. Shakkottai and A. Stolyar, "Scheduling for multiple flows sharing a time-varying channel: The exponential rule," Ann. Math. Statist., vol. 207, pp. 185-202, 2002.

[6] X. Lin and N. Shroff, "Joint rate control and scheduling in multihop wireless networks," Paradise Island, Bahamas, December 2004.

[7] S. Shakkottai, R. Srikant, and A. Stolyar, "Pathwise optimality of the exponential scheduling rule for wireless channels," Adv. Appl. Prob., vol. 36, no. 4, pp. 1021-1045, December 2004.

[8] A. Eryilmaz, R. Srikant, and J. Perkins, "Stable scheduling policies for fading wireless channels," IEEE/ACM Trans. Network., vol. 13, pp. 411424, 2005.

[9] A. Eryilmaz and R. Srikant, "Fair resource allocation in wireless networks using queue-length-based scheduling and congestion control," in Proc. IEEE Infocom., 2005.

[10] M. Neely, E. Modiano, and C. Li, "Fairness and optimal stochastic control for heterogeneous networks," in Proc. IEEE Infocom., vol. 3, Miami, FL, March 2005, pp. 1723-1734.

[11] A. Stolyar, "Maximizing queueing network utility subject to stability: Greedy primal-dual algorithm," Queueing Systems, vol. 50, no. 4, pp. 401-457, August 2005.

[12] X. Lin and S. Rasool, "Constant-time distributed scheduling policies for ad hoc wireless networks," in Proc. Conf. on Decision and Control, 2006.

[13] X. Wu and R. Srikant, "Scheduling efficiency of distributed greedy scheduling algorithms in wireless networks," in Proc. IEEE Infocom., 2006.

[14] A. Eryilmaz, A. Ozdaglar, and E. Modiano, "Polynomial complexity algorithms for full utilization of multi-hop wireless networks," in Proc. IEEE Infocom., 2007.

[15] A. Gupta, X. Lin, and R. Srikant, "Low-complexity distributed scheduling algorithms for wireless networks," in Proc. IEEE Infocom., 2007.

[16] S. Sanghavi, L. Bui, and R. Srikant, "Distributed link scheduling with constant overhead," in Proc. Ann. ACM SIGMETRICS Conf., San Diego, CA, June 2007.

[17] C. Joo, X. Lin, and N. B. Shroff, "Understanding the capacity region of the greedy maximal scheduling algorithm in multi-hop wireless networks," in Proc. IEEE Infocom., Phoenix, Arizona, April 2008.

[18] X. Lin, N. Shroff, and R. Srikant, "A tutorial on cross-layer optimization in wireless networks," IEEE J. Sel. Areas Commun., 2006.

[19] L. Georgiadis, M. J. Neely, and L. Tassiulas, Resource Allocation and Cross-Layer Control in Wireless Networks, 2006, foundations and Trends in Networking.

[20] L. Tassiulas and A. Ephremides, "Throughput properties of a queueing network with distributed dynamic routing and flow control," Advances in Applied Probability, vol. 28, pp. 285 - 307, 1996.

[21] A. Gopalan, C. Caramanis, and S. Shakkottai, "On wireless scheduling with partial channel-state information," in Proc. Ann. Allerton Conf. Communication, Control and Computing, Urbana, IL, September 2007.

[22] X. Qin and R. Berry, "Opportunistic splitting algorithms for wireless networks," in Proc. IEEE Infocom., March 2004.

[23] — , "Opportunistic splitting algorithms for wireless networks with heterogenous users," in Proc. Conf. on Information Sciences and Systems (CISS), March 2004.

[24] S. Adireddy and L. Tong, "Exploiting decentralized channel state information for random access," IEEE Trans. Inform. Theory, no. 2, Feb. 2005.

[25] Z. Ji, Y. Yang, J. Zhou, M. Takai, and R. Bagrodia, "Exploiting medium access diversity in rate adaptive wireless LANs," in Proc. ACM MOBICOM, 2004.

[26] A. Sabharwal, A. Khoshnevis, and E. Knightly, "Opportunistic spectral usage: Bounds and a multi-band CSMA/CA protocol," IEEE/ACM Transactions on Networking, 2006

[27] S. Guha, K. Munagala, and S. Sarkar, "Performance guarantees through partial information based control in multichannel wireless networks," University of Pennsylvania, Tech. Rep., 2006, http://www.seas.upenn.edu/ swati/report.pdf.

[28] D. Zheng, W. Ge, and J. Zhang, "Distributed opportunistic scheduling for ad-hoc communications: An optimal stopping approach," in ACM Mobihoc, 2007.
[29] N. Chang and M. Liu, "Optimal channel probing and transmission scheduling for opportunistic spectrum access," in ACM Int. Conf. on Mobile Computing and Networking (MobiCom), September 2007.

[30] K. Kar, X. Luo, and S. Sarkar, "Throughput-optimal scheduling in multichannel access point networks under infrequent channel measurements," in Proceedings of IEEE Infocom, Anchorage, AK, May 2007.

[31] A. Pantelidou, A. Ephremides, and A. L. Tits, "Joint scheduling and routing for ad-hoc networks under channel state uncertainty," in 5th Intl. Symposium on Modeling and Optimization in Mobile, Ad-Hoc and Wireless Networks (WiOpt), April 2007.

[32] L. Ying and S. Shakkottai, "On throughput optimality with delayed network-state information," in Technical Report, 2008.

\section{Appendix A: Proof of Theorem 2}

We define a Lyapunov function such that

$$
V[t]=\sum_{l \in \mathcal{L}} Q_{l}^{2}[t]
$$

First, it can be shown that there exists $B>0$, which is independent of $\mathbf{Q}$ such that

$$
\begin{aligned}
& \left.\mathbf{E}\left[V[t+1]-V[t] \mid \mathbf{J}[t-\tau], \mathbf{Q}\left[t-\tau_{q}\right]\right]\right] \\
\leq \quad & B+2 \sum_{l \in \mathcal{L}} Q_{l}\left[t-\tau_{q}\right] \times \\
& \left(\mathbf{E}\left[\boldsymbol{\lambda}_{l}[t]\right]-\mathbf{E}\left[C_{l}[t] \Phi_{l}^{*}[t] \mid \mathbf{J}[t-\tau]\right], \mathbf{Q}\left[t-\tau_{q}\right]\right),
\end{aligned}
$$

where

$$
\begin{aligned}
\Phi_{l}^{*}[t]= & 1_{C_{l}[t] \geq \Theta_{l}^{*}\left(S_{l}[t], R_{l}[t]\right)} \times \\
& \prod_{j \neq l}\left(\begin{array}{ll}
1-1 & \begin{array}{c}
\left|S_{j}[t]-R_{l}[t]\right| \leq(1+\Delta) D, \\
C_{j}[t] \geq \Theta_{j}^{*}\left(S_{j}[t], R_{j}[t]\right)
\end{array}
\end{array}\right),
\end{aligned}
$$

and $\Theta_{j}^{*}\left(S_{j}[t], R_{j}[t]\right)$ is the optimal solution to problem (2).

Now given that $(1+\epsilon) \mathbf{E}[\boldsymbol{\lambda}[t]] \in \Lambda_{\tau}$, according to the definition of $\Lambda_{\tau}$, there exists a set of $\eta_{\{\mathbf{c},(\mathbf{s}, \mathbf{r})\}} \in \Lambda_{\{\mathbf{c},(\mathbf{s}, \mathbf{r})\}}$ such that

$$
(1+\epsilon) \mathbf{E}[\boldsymbol{\lambda}[t]]=\sum_{\{\mathbf{c},(\mathbf{s}, \mathbf{r})\}} \pi_{\{\mathbf{c},(\mathbf{s}, \mathbf{r})\}} \eta_{\{\mathbf{c},(\mathbf{s}, \mathbf{r})\}} .
$$

Assuming that $\mathbf{J}[t-\tau]=\{\mathbf{c},(\mathbf{s}, \mathbf{r})\}$, we have that

$$
\begin{gathered}
\left.\mathbf{E}\left[V[t+1]-V[t] \mid\{\mathbf{c},(\mathbf{s}, \mathbf{r})\}, \mathbf{Q}\left[t-\tau_{q}\right]\right]\right] \\
\leq \quad B+2 \sum_{l \in \mathcal{L}} Q_{l}\left[t-\tau_{q}\right]\left(\mathbf{E}\left[\boldsymbol{\lambda}_{l}[t]\right]-\eta_{\{\mathbf{c},(\mathbf{s}, \mathbf{r})\}}\right) \\
+2 \sum_{l \in \mathcal{L}} Q_{l}\left[t-\tau_{q}\right] \eta_{\{\mathbf{c},(\mathbf{s}, \mathbf{r})\}} \\
-2 \sum_{l \in \mathcal{L}} Q_{l}\left[t-\tau_{q}\right] \mathbf{E}\left[C_{l}[t] \Phi_{l}^{*}[t] \mid\{\mathbf{c},(\mathbf{s}, \mathbf{r})\}\right] .
\end{gathered}
$$

According to the definition of $\mathbf{L}$, it is easy to see that the optimization problem (2) can be re-written as

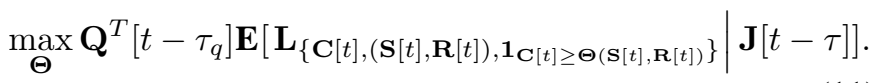

Also we have that

$$
\begin{gathered}
\boldsymbol{\eta}_{\{\mathbf{c},(\mathbf{s}, \mathbf{r})\}} \in \Lambda_{\{\mathbf{c},(\mathbf{s}, \mathbf{r})\}}= \\
\mathcal{C} \mathcal{H}_{\Theta}\left(\mathbf{E}\left[\mathbf{L}_{\left\{\mathbf{C}[t],(\mathbf{S}[t], \mathbf{R}[t]), \mathbf{1}_{\mathbf{C}[t] \geq \Theta(\mathbf{S}[t], \mathbf{R}[t])\}} \mid\right.} \mid \mathbf{J}[t-\tau]\right]\right) .
\end{gathered}
$$

Thus, we have that

$$
\mathbf{Q}^{T}\left[t-\tau_{q}\right] \boldsymbol{\eta}_{\{\mathbf{c},(\mathbf{s}, \mathbf{r})\}} \leq(11)
$$


which implies that

$$
(9) \leq(10)
$$

holds for all $\{\mathbf{c},(\mathbf{s}, \mathbf{r})\}$. Furthermore, $\{\mathbf{C}[t-\tau], \mathbf{S}[t-\tau], \mathbf{R}[t-$ $\tau]\}$ is independent of $\mathbf{Q}\left[t-\tau_{q}\right]$ since $\tau \leq \tau_{q}$. Thus, we can conclude that

$$
\begin{aligned}
& \mathbf{E}\left[V[t+1]-V[t] \mid \mathbf{Q}\left[t-\tau_{q}\right]\right] \\
& =\sum_{\{\mathbf{c},(\mathbf{s}, \mathbf{r})\}} \pi_{\{\mathbf{c},(\mathbf{s}, \mathbf{r})\}} \mathbf{E}\left[V[t+1]-V[t] \mid\{\mathbf{c},(\mathbf{s}, \mathbf{r})\}, \mathbf{Q}\left[t-\tau_{q}\right]\right] \\
& \leq B+2 \sum_{l \in \mathcal{L}} Q_{l}\left[t-\tau_{q}\right] \times \\
& \\
& \quad\left(\mathbf{E}\left[\lambda_{l}[t]\right]-\sum_{\{\mathbf{c},(\mathbf{s}, \mathbf{r})\}} \pi_{\{\mathbf{c},(\mathbf{s}, \mathbf{r})\}} \eta_{\{\mathbf{c},(\mathbf{s}, \mathbf{r})\}}\right) \\
& \leq B-2 \epsilon \sum_{l \in \mathcal{L}} Q_{l}\left[t-\tau_{q}\right] \mathbf{E}\left[\lambda_{l}[t]\right],
\end{aligned}
$$

where the last inequality holds due to (8). Now the theorem follows from the Foster's Criterion.

\section{Appendix B: Proof of TheOREM 3}

We consider the virtual network and let $\tilde{\mathbf{Q}}[t]$ denote the queue-lengths of the network system. We define a Lyapunov function such that

$$
V[t]=\sum_{l \in \mathcal{L}} \tilde{Q}_{l}^{2}[t]
$$

First, it can be shown that there exists $B>0$, which is independent of $\tilde{\mathbf{Q}}$ such that

$$
\begin{aligned}
& \quad \mathbf{E}\left[V[t+1]-V[t] \mid \mathbf{J}[t-\tau], \tilde{\mathbf{Q}}\left[t-\tau_{q}\right]\right] \\
& \leq \quad B+2 \sum_{l \in \mathcal{L}} \tilde{Q}_{l}\left[t-\tau_{q}\right] \times \\
& \quad\left(\mathbf{E}\left[\lambda_{l}[t]-C_{l}[t] \tilde{\Phi}_{l}[t] \mid \mathbf{J}[t-\tau]\right], \tilde{\mathbf{Q}}\left[t-\tau_{q}\right]\right),
\end{aligned}
$$

where $\tilde{\boldsymbol{\Phi}}$ is defined similar as (7).

Now given that

$$
(1+\epsilon)\left(\mathbf{E}[\lambda[t]]+C_{\max }\left(1-\left(\frac{1}{2 \frac{W}{X}+1}\right)^{2}\right) \mathbf{1}\right) \in \Lambda_{\tau},
$$

there exists a set of $\eta_{\{\mathbf{c},(\mathbf{s}, \mathbf{r})\}} \in \Lambda_{\{\mathbf{c},(\mathbf{s}, \mathbf{r})\}}$ such that

$$
\begin{aligned}
& \left((1+\epsilon)\left(\mathbf{E}[\boldsymbol{\lambda}[t]]+C_{\max }\left(1-\left(\frac{1}{2 \frac{W}{X}+1}\right)^{2}\right) \mathbf{1}\right)\right. \\
= & \sum_{\{\mathbf{c},(\mathbf{s}, \mathbf{r})\}} \pi_{\{\mathbf{c},(\mathbf{s}, \mathbf{r})\}} \eta_{\{\mathbf{c},(\mathbf{s}, \mathbf{r})\} .}
\end{aligned}
$$

Assuming that $\mathbf{J}[t-\tau]=\{\mathbf{c},(\mathbf{s}, \mathbf{r})\}$, we have that

$$
\begin{aligned}
& \mathbf{E}\left[V[t+1]-V[t] \mid\{\mathbf{c},(\mathbf{s}, \mathbf{r})\}, \tilde{\mathbf{Q}}\left[t-\tau_{q}\right]\right] \\
\leq \quad & B+2 \sum_{l \in \mathcal{L}} \tilde{Q}_{l}\left[t-\tau_{q}\right] \times \\
& \left(\mathbf{E}\left[\boldsymbol{\lambda}_{l}[t]+C_{\max } 1_{S_{l}[t] \in \mathcal{C}^{i}} \mid S_{l}[t-\tau]=s_{l}\right]-\eta_{\{\mathbf{c},(\mathbf{s}, \mathbf{r})\}}\right) \\
+ & 2 \sum_{l \in \mathcal{L}} \tilde{Q}_{l}\left[t-\tau_{q}\right] \eta_{\{\mathbf{c},(\mathbf{s}, \mathbf{r})\}} \\
& -2 \sum_{l \in \mathcal{L}} \tilde{Q}_{l}\left[t-\tau_{q}\right] \mathbf{E}\left[C_{l}[t] \tilde{\Phi}_{l}[t] \mid\{\mathbf{c},(\mathbf{s}, \mathbf{r})\}, \tilde{\mathbf{Q}}\left[t-\tau_{q}\right]\right] .
\end{aligned}
$$

Let $\Theta^{*}$ denote the optimal decision policy in the original network with the complete delayed network information, we have that

$$
\begin{aligned}
& \sum_{l \in \mathcal{L}} \tilde{Q}_{l}\left[t-\tau_{q}\right] \eta_{\{\mathbf{c},(\mathbf{s}, \mathbf{r})\}} \\
\leq & \sum_{l \in \mathcal{L}} \tilde{Q}_{l}\left[t-\tau_{q}\right] \mathbf{E}\left[C_{l}[t] \Phi_{l}^{*}[t] \mid\{\mathbf{c},(\mathbf{s}, \mathbf{r})\}, \tilde{\mathbf{Q}}\left[t-\tau_{q}\right]\right] \\
\leq & \sum_{l \in \mathcal{L}} \tilde{Q}_{l}\left[t-\tau_{q}\right] \mathbf{E}\left[C_{l}[t] \tilde{\Phi}_{l}[t] \mid\{\mathbf{c},(\mathbf{s}, \mathbf{r})\}, \tilde{\mathbf{Q}}\left[t-\tau_{q}\right]\right] .
\end{aligned}
$$

Furthermore, $\{\mathbf{C}[t-\tau], \mathbf{S}[t-\tau], \mathbf{R}[t-\tau]\}$ is independent of $\tilde{\mathbf{Q}}\left[t-\tau_{q}\right]$ since $\tau \leq \tau_{q}$. Thus, we can conclude that

$$
\begin{aligned}
& \mathbf{E}\left[V[t+1]-V[t] \mid \tilde{\mathbf{Q}}\left[t-\tau_{q}\right]\right] \\
& =\sum_{\{\mathbf{c},(\mathbf{s}, \mathbf{r})\}} \pi_{\{\mathbf{c},(\mathbf{s}, \mathbf{r})\}} \mathbf{E}\left[V[t+1]-V[t] \mid\{\mathbf{c},(\mathbf{s}, \mathbf{r})\}, \tilde{\mathbf{Q}}\left[t-\tau_{q}\right]\right] \\
& \leq B+2 \sum_{l \in \mathcal{L}} \tilde{Q}_{l}\left[t-\tau_{q}\right]\left(\mathbf{E}\left[\boldsymbol{\lambda}_{l}[t]\right]+\right. \\
& \left.C_{\max } \sum_{\mathbf{s}_{l} \in \mathcal{C}^{i}} \pi_{\{\mathbf{c},(\mathbf{s}, \mathbf{r})\}}-\sum_{\{\mathbf{c},(\mathbf{s}, \mathbf{r})\}} \pi_{\{\mathbf{c},(\mathbf{s}, \mathbf{r})\}} \eta_{\{\mathbf{c},(\mathbf{s}, \mathbf{r})\}}\right)
\end{aligned}
$$

Note that

$$
\sum_{\{\mathbf{c},(\mathbf{s}, \mathbf{r})\}: \mathbf{s}_{l} \in \mathcal{C}^{i}} \pi_{\{\mathbf{c},(\mathbf{s}, \mathbf{r})\}}=1-\left(\frac{X}{2 W+X}\right)^{2} .
$$

Thus, we can conclude the theorem holds because

$$
\begin{aligned}
& \mathbf{E}\left[V[t+1]-V[t] \mid \tilde{\mathbf{Q}}\left[t-\tau_{q}\right]\right] \\
& =\sum_{\{\mathbf{c},(\mathbf{s}, \mathbf{r})\}} \pi_{\{\mathbf{c},(\mathbf{s}, \mathbf{r})\}} \mathbf{E}\left[V[t+1]-V[t] \mid\{\mathbf{c},(\mathbf{s}, \mathbf{r})\}, \tilde{\mathbf{Q}}\left[t-\tau_{q}\right]\right] \\
& \leq B-2 \epsilon \sum_{l \in \mathcal{L}} \tilde{Q}_{l}\left[t-\tau_{q}\right] \times \\
& \left(\mathbf{E}\left[\lambda_{l}[t]\right]+C_{\max }\left(1-\left(\frac{X}{2 W+X}\right)^{2}\right)\right)
\end{aligned}
$$

